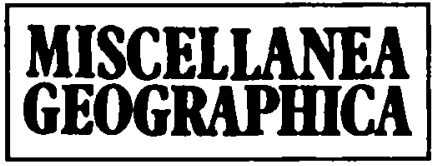

WARSZAWA 1992 Vol. 5

Izabella Łęcka

\title{
TRANSFORMATIONS DE L'ENVIRONNEMENT NATUREL ASSOCIÉES AUX GRANDS OUVRAGES HYDRAULIQUES ET LEUR DÉPENDENCE DU CLIMAT
}

Les ouvrages hydrauliques engendrent d'importantes transformations de l'environnement naturel de l'homme. Certains chercheurs avancent même que l'influence de constructions hydrauliques dépasse l'ingérence de grands établissements industriels, bien que celle-ci ne soit pas des moindres (Głodek 1985).

De grands ouvrages hydrauliques sont élevés partout dans le monde, aussi bien dans les zones tempérées que sous les tropiques. Et on évoque de plus en plus souvent, surtout dès les années quatre-vingt, des effets nocifs accompagnant ces ouvrages. A mon avis, pourtant, on ne s'intéresse pas suffisamment à l'interdépendance de ces transformations et du climat, et surtout du degré de l'humidité de ce dernier.

Les différences entre les effets associés aux constructions hydrauliques fonctionnant sous le climat tempéré et ceux que l'on observe sous le climat chaud, semblent être évidentes. Parmi les plus importantes, citons des transformations climatiques, vitesse de l'eutrophisation, structure générique des végétaux aquatiques et marécageux, absence, sous le climat tempéré, des maladies liées directement avec des migrations de populations, lesdites migrations y étant peu importantes (le territoire de l'Union Soviétique exclu). Le climat tempéré semble plus favorable étant donné le nombre moins important d'organismes vivants nuisibles à la végétation donc plus faciles à détruire. En ce qui concerne les forêts de la zone tempérée, leur dégradation entraîne moins de pertes parmi les espèces de plantes et d'animaux que dans le cas de la dégradation de la forêt dense équatoriale.

Sous le climat chaud, les différences entre les influences exercées par de grands projets hydrauliques sur l'environnement naturel sont moins évidentes et résultent, en général, de la moyenne annuelle des précipitations différente dans divers points de cette région. La plupart de travaux traitant de l'influence de grands barrages hydrauliques sur les conditions naturelles sous le climat chaud considèrent cette zone en tant qu'un tout et n'indiquent pas de traits caractéristiques propres à un climat humide ou à un climat sec. Ainsi, on pourrait 
se demander si les différences en question existent réellement, donc le sujet semble suffisamment intéressant et digne d'être examiné plus profondément.

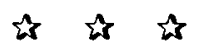

Les grands barrages et les grands lacs de retenue qui les accompagnent (dont le volume dépasse 100 millions de mètres cubes) sont construits avant tout sous les climats tempérés (42\%), ensuite dans la zone sous-tropicale (36\%); il y en a moins dans la zone tropicale $(8 \%)$ et sous-équatoriale (11\%), et très peu sous les climats équatorial et polaire (1,5\% dans les deux cas). Les objectifs économiques à atteindre dépendent à un degré important du climat sous lequel fonctionnent les ouvrages en question. Dans la zone aride, ces objectifs sont les suivants: irrigation, régularisation des débits, protection contre les inondations, production d'énergie électrique,enfin pêche et navigation. En Algérie, p.ex.,plus de $90 \%$ de lacs de barrage travaillent pour les besoins de l'irrigation. Il en est autrement dans la zone humide où la tâche principale consiste à produire de l'énergie électrique - c'est le cas des projets hydrauliques en Amazonie.

Un lac de barrage est rentable lorsqu'il accumule l'écoulement de plusieurs années, indépendamment du climat. Ainsi, l'absence d'un terrain propre à retenir les eaux s'avère souvent ce facteur qui rend impossible la réalisation d'un projet. L'accumulation de l'écoulement de plusieurs années ou, dans le pire cas, d'une seule année, est particulièrement importante sous le climat sec vu la nécessité de balancer les débits des années sèches et des années humides soit les débits de la saison sèche et de la saison des pluies.Ainsi, p.ex., le lac Nasser retient l'écoulement du Nil de 18 mois, tandis que les lacs Kossou et Kainji situés dans une région beaucoup plus humide - seulement celui de 9 mois, sans parler d'un lac de retcnue dans le bassin du Parana qui en retient encore moins (Głodek 1985).

Les réservoirs plus importants sont censés servir plus longtemps, le processus d'envasement y étant plus lent. Les sédiments se déposent le plus vite dans les lacs de barrage situés dans des terrains montagneux, et cela indépendamment du climat. Les fleuves et rivières de la zone aride retiennent plus de matériaux alluviaux que ceux et celles traversant les régions humides.

Quant à l'eutrophisation, certains chercheurs (Goldsmith, Hildyard 1984) sont d'avis que ce processus concerne, en premier lieu, les régions chaudes et sèches. Ils illustrent leur thèse avec le cas de l'Égypte où jusqu'en 1974, des végétaux aquatiques ont envahi $80 \%$ des eaux du lac Nasser. Néanmoins, cette opinion semble inexacte car l'eutrophisation du fleuve Zaïre, du bassin de l'Amazone et celle du Suriname se faisaient à une allure semblable - la dégradation des 50\% des eaux du réservoir à Brokopendo s'est effectuée en 2 ans (Cummings 1990). L'évaporation sur la surface de l'eau du réservoir dépend du degré d'humidité de l'air, étant donné que la température dans toute la région concernée est élevée. Ainsi, le lac Nasser perd env. $15,5 \mathrm{~km}^{3}$ d'eau par an tandis que le lac Volta, situé sous un climat beaucoup plus humide en perd seulement $12,5 \mathrm{~km}^{3}$ avec le 
volume moins grand de $20 \mathrm{~km}^{3}$ et la superficie dépassant d'un tiers celle du lac Nasser (Glodek 1985). Les pertes en eau dues à l'évaporation s'élèvent à 3000 $\mathrm{mm}$ par an dans les régions sèches de l'Inde contre $1400 \mathrm{~mm}$ env. dans la Guyane humide (Goldsmith, Hildyard 1984). Une forte évaporation sur les surfaces respectives de l'eau et du sol fait que le processus de salinisation aussi bien de l'une que de l'autre est plus rapide et plus intense sous un climat aride que sous un climat humide.

Tandis que le bassin de retenue se comble progressivement, le niveau de la nappe phréatique change sur une vaste superficie. Dans le cas des terrains humides, la remontée de la nappe phréatique entraîne une altération des conditions de la culture des céréales, les forêts et les surfaces herbeuses risquent d'être noyées. La prévention de ces phénomènes est à la fois difficile et coûteuse. Dans les terrains arides, la nappe phréatique relevée assure des meilleures conditions pour l'agriculture.

L'irrigation pendant toute l'année et la remontée de la nappe phréatique font augmenter le degré d'humidité de l'air et créent ainsi les conditions idéales au développement des parasites vecteurs des maladies telles que paludisme, bilharziose, onchocercose, etc. Chez l'homme, le paludisme est provoqué par l'une des quatre variétés des plasmodies. Pour devenir actif, le plasmodium falciparum variété la plus fréquente - réclame la température au-dessus de $19^{\circ} \mathrm{C}$ et l'humidité dépassant $52 \%$. C'est ainsi que le paludisme s'est manifesté dans des terrains arides, jusque-là non contaminés, où l'on venait d'installer l'irrigation artificielle fonctionnant toute l'année.Le plasmodium s'attaque aux populations locales non immunisées contre cette maladie. Dans des terrains humides, la différence avant et après la construction d'un barrage n'est pas visible - le paludisme y sévissant toujours, dans ce domaine rien n'a changé. Soulignons tout de même que dans les deux cas - aussi bien sous les climats secs que sous les climats humides, la vitesse de la propagation de cette maladie est également grande, ce qui est dû surtout aux mouvements migratoires accompagnant l'offre de nouveaux emplois à la construction d'un barrage.

La diffusion de la bilharziose est un problème plus compliqué vu que son développement ne dépend pas seulement de l'humidité et de la température mais également du chimisme de l'eau.On a observé qu'il y a des territoires où la bilharziose ne se manifeste pas en dépit de la température et du degré d'humidité élevés durant toute l'année. C'est, entre autres, le cas de l'Amazonie. Aujourd'hui, pourtant, les réservoirs de retenue nouvellement installés ont rapidement changé le chimisme de l'eau de sorte que dans certains endroits, on a noté l'apparition des mollusques transmetteurs de cette maladie (Goldsmith, Hildyard 1984).

La construction d'ouvrages hydrauliques dans la zone équatoriale humide est associée à la dégradation de la forêt dense humide dont la biocénose est plus riche que celle d'autres terrains. La forêt dense humide n'occupant que $7 \%$ de la surface de la Terre, elle est pourtant peuplée de $40 \%$ de toutes les espèces aussi bien végétales qu'animales (éd. Brown 1990). La destruction des forêts entraîne 
non seulement une diminution de richesses génétiques; en même temps disparaît une abondante source d'aliments. On estime que les ongulés qui dépérissent pourraient assurer la quantité de protéines égale ou même plus grande que les poissons pêchés dans les eaux d'un lac de retenue. En même temps, le fait de transformer les terrains boisés en des terres cultivables accélère la vitesse de la dénudation des sols.

Lorsqu'un réservoir de barrage est comblé sans être auparavant déblayé, débarrassé d'arbres, d'arbustes et d'herbes, non seulement il n'est pas navigable mais la qualité de ses eaux est inférieure. Sous un climat aride, ce phénomène se manifeste à une échelle moins importante.

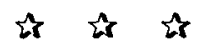

Les différences entre divers effets négatifs accompagnant les grands ouvrages hydrauliques sont évidentes et dépendent du degré d'humidité du climat chaud donc l'examen plus poussé de ce problème semble nécessaire. Une étude entreprise dans ce but pourrait d'un côté définir le type de climat où les effets nocifs sont moins nombreux ou moins importants, et de l'autre - mettre au point des méthodes de minimiser lesdits effets. En ce qui concerne les régions humides, une étude de ce genre pourrait répondre à la question si la production d'énergie électrique aux frais écologiques très élevés s'avérerait réellement rentable dans le bilan général.

\section{BIBLIOGRAPHIE}

B r ow n, L.R. (éd.), 1990, State of the World (édition polonaise), PWE, Warszawa.

Cu m ming s, B.J., 1990, Dam the Rivers Damn the People, Earthscan Publications Ltd., London.

Glodek, J., 1985, Jeziora zaporowe świata (Les lacs de retenue dans le monde), PWN, Warszawa.

Golds mith, E., Hildyard, N., 1984, "The Social and Environmental Effects of Large Dams, vol.1, ECOROPA. 